\title{
Controlled exchange of metallic cations by a polypyrrole-based resin
}

\author{
Jérôme C[1], Martinot L[2], Jerome R[1], Strivay D[3], Weber G[3]
}

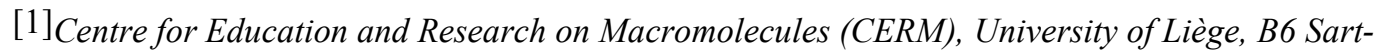
Tilman, 4000 Liège, Belgium

${ }^{[2]}$ Radiochemistry, University of Liège, B16 Sart-Tilman, 4000 Liège, Belgium

[3] Nuclear Physics, University of Liège, B15 Sart-Tilman, 4000 Liège, Belgium

\section{Résumé}

Ces travaux montrent que la fixation et le relargage des cations $\mathrm{Cs}^{+}, \mathrm{Ba}^{2+}, \mathrm{La}^{3+}$ et $\mathrm{Th}^{4+}$ peuvent être contrôlés par l'application d'un potentiel à une résine de polypynole dopée au polystyrène sulfonate. Des mesures radioméuiques (comptage a) et nucléaires (rétrodiffusion de Rutherford) ont permis de caractériser la réversibilité du procédé, notamment en fonction de la charge de l'ion échangé.

\section{Mots-clés}

échangeurs d'ions, polypynole, cation métallique, contrôle électrochimique.

\begin{abstract}
This work shows that the binding and the release of $\mathrm{Cs}^{+}, \mathrm{Ba}^{2+}, \mathrm{La}^{3+}$ and $\mathrm{Th}^{4+}$ cations can be controlled by the application of a potential to a polypy $\pi$ ole doped with polystyrene sulfonate exchangeresin. Radiometric and nuclear techniques were carried out to characterize the reversibility of the process in relation with the exchanged-cation charge.
\end{abstract}

\section{Key words}

ions-exchange resin, polypy $\pi$ ole, metallic cation, electrochemical control.

\section{Introduction}

Within the prospect of water treatment, we have studied a new type of exchange resin characterized by its ability to control the ion exchange via the applied potential Polypyrrole (PPy) doped with a polyelectrolyte (polystyrene sulfonate (PSSO3)) constitutes the cationic resin [1] used to study the exchange of various metal cations such as $\mathrm{Cs}^{+}, \mathrm{Ba}^{2+}, \mathrm{La}^{3+}, \mathrm{Th}^{4+}$

\section{Experimental}

$\mathrm{PPy} / \mathrm{PSSO}_{3}$ is potentiostatically synthesized $(\mathrm{E}=+07 \mathrm{~V} / \mathrm{SCE})$ in a $0 . .1 \mathrm{M}$ pyrrole / " $0.1 \mathrm{M} \mathrm{PSS} 0_{3} \mathrm{Na}$ (MW=70000,Aldrich) aqueous solution onto carbon or platinum electrodes The electrodeposited films are rinsed with twice-distilled water and investigated in decimolar solutions of ThCI $4, \mathrm{LaCl}_{3}, \mathrm{BaCl}_{2}$, and $\mathrm{CsCl}$ using an M270 EG\&G PAR potentiostat/galvanostat Samples characterization is achieved using an $\alpha$ counter in the case of radioactive thorium Otherwise, Rutherford Backscattering technique (RBS) [2] and scanning electron microscopy (SEM) coupled with electron diffraction X-ray analysis (EDXA) are used to detect non-radioactive cations.

\section{Results and discussion}

Cyclic voltammetry evidences the well-defined reduction and reoxidation peaks of the polymer resin whatever the cation present in the medium.. No electrochemical reaction of the cation to be fixed occurs during the polymer reduction since all the above quoted cations are characterized by only one stable oxidation state in water. This constitutes the first requisite condition for the application of the electrochemical controiled-exchange process.

In solutions containing $\mathrm{Cs}^{+} \mathrm{Ba}^{2+}$, or $\mathrm{La}^{3+}$ two successive scans are superimposed, 
demonstrating the reproducibility of the polymer redox process Moreover, the relation $\mathrm{i}=\mathrm{f}(\mathrm{v})$ is linear, depicting the usual behaviour observed for species that are precipitated on the electrode surface like conducting polymers In the case of the ThCL4 solution, an alteration of the PPy redox cycle is evidenced by the intensities of both the reduction and oxidation peaks which decrease with repetitive scans. The exchange of $\mathrm{Th}^{4+}$ should thus not be totally reversible.

The effect of the reduction current quantity (Qr) on the amount of fixed cations is then evaluated. According to the fixation process, we can observe a linear evolution between the amount of incorporated Th (determined by $\alpha$ counting) and the degree of reduction of the film. In this particular case, we have also shown that the radioactive fixed species appears to be a trivalent cation, presumably $[\mathrm{ThCl}]^{3+}$ or $[\mathrm{ThOH}]^{3+}$. Likewise, the RBS technique has also shown that the more the polymer film is reduced, the more the amount of fixed lanthanum is important. Thus the binding of the cations depends on the potential applied to the resin.

The reversibility of the process is also evaluated for each cation type, $\alpha$-counting of a reduced and reoxidized film in a ThCL4 solution shows that $60 \%$ of the fixed Th can be released during this first scan. This confirms the loss of the reversibility already observed by cyclic voltammetry

The RBS spectra of reduced and reoxidized films in $\mathrm{LaCl}_{3}$ and in $\mathrm{BaCl}_{2}$ aqueous solutions allow us to characterize both the binding and the release of these cations From the lowest to the highest energy of the back-scattering spectrum, we can observe all the characteristic peaks of the elements of the polymer: $\mathrm{C}, \mathrm{N}, \mathrm{O}$, and $\mathrm{S}$ followed by $\mathrm{CI}$ and La or Ba peaks depending on the atoms incorporated during the film reduction. Comparing the half-widths of the $\mathrm{S}$ and $\mathrm{La}$ or Ba peaks, we can conclude to the presence of a superficial binding of both $\mathrm{La}^{3+}$ and $\mathrm{Ba}^{2+}$. They remain concentrated nearby the film surface with a penetrating depth of $0.5 \mu \mathrm{m}$ for La and $0 . .25 \mu \mathrm{m}$ for $\mathrm{Ba}$; they diffuse inside the polymer following a linear decreasing profile.

The ratios $\mathrm{S} / \mathrm{La}=1 / 0.02$ and $\mathrm{S} / \mathrm{Ba}=1 / 0.06$ have been determined from the area of the respective peaks: from these results it appears that an important amount of "passive" sulfonate functions are incorporated in the polymer film The lower incorporation of $\mathrm{La}$, as compared to $\mathrm{Ba}$, can be partially explained by the difference of the charge of the two cations.

We can also observe that the La and the Ba peaks are still present after reoxidation of the resin: the release is thus not complete. Moreover, we are able to release $85 \%$ of the fixed La. On the ieoxidized film, a ratio $\mathrm{La} / \mathrm{Cl}=1 / 2$ was calculated, meaning that some amount of the La remains trapped in the polymer film as a counterion of the . sulfonate functions, "passive" sulfonate sites compensating the positive charges on the ieoxidized PPy backbone. On the other hand, $50 \%$ of the fixed $\mathrm{Ba}$ is released and a ratio $\mathrm{Ba} / \mathrm{Cl}=1 / 2$ has been measured This fact corresponds to the formation of $\mathrm{BaCl}_{2}$ on the surface of the polymer. Indeed, when an oxidizing potential is applied to the resin, the $\mathrm{Ba}^{2+}$ is released near the electrode surface and this causes an increase in the local concentration of this cation and leads to the precipitation of the chloride salt onto the electrode The same results were observed for the exchange of the Cs cation. The SEM/EDAX analysis of the reoxidized polymer resin singles out a ratio $\mathrm{Cs} / \mathrm{Cl}=: 1$ while only $\mathrm{Cs}$ atoms without any $\mathrm{CI}$ are detected on a reduced resin.

We can thus conclude that $\mathrm{Cs}^{+}$and $\mathrm{Ba}^{2+}$ binding and release are reversible but contamination by the chloride salt occurs during the release process. When the fixed species are $\mathrm{La}^{3+}$ or $\mathrm{Th}^{4+}$, the release process is incompleted and some cations remain in the polymer film. The influence of the releasing medium on the reversibility of the system is under consideration, notably using nitrate salts to avoid the precipitation of the chlorides.

\section{References}

1 Momma T, Nishimura K, Osaka T (1994) J. Electrochem, Soc. 141,2326

2 Chu W, Mager J, Nicolet M (1978) Backscattering Spectrometry, Academic Press, New York. 